\title{
A Box-Cox Approach to QT Interval Correction for Heart Rate
}

\author{
Duolao Wang, $\mathrm{PhD}^{1 *}$, Radivoj Arezina, $\mathrm{MD}^{2}$, Jorg Taubel, $\mathrm{MD}^{2}$ \\ ${ }^{1 *}$ Department of Clinical Sciences, Liverpool School of Tropical Medicine \\ ${ }^{2}$ Richmond Pharmacology, St George's University of London
}

\begin{abstract}
QT interval on an electrocardiogram (ECG) trace is a widely used surrogate parameter in drug development to detect the potential of drugs to cause life threatening cardiac arrhythmias. It changes inversely with the magnitude of heart rate (Heart rate $=60 / \mathrm{RR}$, RR stands for RR interval on an ECG reading) and is often corrected to a heart rate independent value known as the corrected QT interval (QTc). Various parametric QT correction formulae have been proposed by specifying the QT and heart rate relationships explicitly by a fixed number of parameters. This paper proposes to use the Box-Cox transformation to fit the QTRR relationship, and develops a new method for correcting the QT interval for heart rate. The six commonly used parametric models are the special cases of Box-Cox transformed model. We discuss the model parameter estimation and assess the performance of QT correction formulae derived from four types of Box-Cox transformations using four off-drug ECG datasets and one on-drug ECG dataset, as well as a simulated dataset. The results show that all four derived QT correction formulae from Box-Cox transformation generate a heart rate independent QTc and that the QT correction formula derived from transformation of both QT and RR generates QTc with smaller variations. The Box-Cox transformation represents a very flexible family for modelling QT-RR relationships including the six commonly used parameter models, thus providing a potentially better QT correction method than the existing parametric models.
\end{abstract}

Key words: ECG; Box-Cox transformation; QTc; QT interval correction; horough QT study.

\section{Introduction}

QT interval on an electrocardiogram (ECG) trace (Goldenberg et al., 2006), representing electrical depolarization and repolarization of the ventricles, is an important and widely used surrogate parameter to identify drugs that have the potential to cause life threatening cardiac arrhythmias. Prolongation of cardiac repolarisation is recognised to be associated with various serious arrhythmias, e.g. Torsade de Point (TdP) and this has thus become an important safety issue attracting a great deal of attention from both regulatory agencies and the pharmaceutical industry (ICH, 2005).

\footnotetext{
${ }^{*}$ Corresponding author.
} 
Over the past decade, a number of drugs have been shown to prolong cardiac repolarisation and this effect has become an important safety issue attracting a great deal of attention from both regulatory agencies and the pharmaceutical industry. Since the 1990's, new and existing drugs have been subjected to close scrutiny for their potential adverse effect on QT. This was in response to the finding that terfenadine, the first and very popular non-sedating antihistamine for therapeutic use, caused numerous cases of sudden death and TdP in association with prolonged QT interval. At the time the drug manufacturer was considering a regulatory application for terfenadine to become a non-prescription medication available over the counter. However, due to its effect on QTc, terfenadine was withdrawn from the market in 1997.

The QT interval changes inversely with the magnitude of heart rate (Heart rate $(H R)=60 / R R, R R$ stands for RR interval on an ECG reading) and is often corrected to a heart rate independent value known as the corrected QT interval (QTc), which is widely used in clinical practice to assess drug safety (Malik 2001; ICH, 2005; Goldenberg et al., 2006). The QTc interval represents the QT interval at a standardised heart rate of 60/minute. The goal of heart rate correction is to provide QTc interval values that are independent of the corresponding RR interval values.

Deriving a QT correction formula usually involves two steps. The first step is to fit a regression model with unknown parameters to describe the QT-RR relationship. For example, we assume that there is a linear relationship between QT and RR, e.g. $Q T=\beta+\alpha R R$, where $(\beta, \alpha)$ can be estimated using the least square method. The second step is to derive a correction formula based the fitted model. This is done by canceling out the parameter $\beta$ using the condition that $\mathrm{QTc}=\mathrm{QT}$ when $\mathrm{RR}=1$. For the above example, we have $Q T c=Q T+\alpha(1-R R)$.

Various statistical models have been proposed and developed to fit QT-RR relationships (ICH, 2005; Goldenberg et al., 2006). Six most commonly used regression models are used to fit the QT-RR relationships (Bazett, 1920; Fridericia, 1920; Hodges et al., 1983; Malik et al., 2002; Sagie et al., 1992; Goldenberg et al., 2006; Wang et al., 2012)

Model 1: Linear model

$$
Q T=\beta+\alpha R R
$$

Model 2: Hyperbolic model

$$
Q T=\beta+\alpha / R R
$$

Model 3: Parabolic model

$$
Q T=\beta R R^{\alpha}
$$

Model 4: Logarithmic model

$$
Q T=\beta+\alpha \ln (R R)
$$


Model 5: Shifted logarithmic model

$$
Q T=\ln (\beta+\alpha R R)
$$

Model 6: Exponential model

$$
Q T=\beta+\alpha e^{-R R}
$$

Each of the above six regression models has two parameters: $\alpha$ and $\beta$. Once they are estimated, they can then be converted to the following generic heart rate correction formulae using the condition $Q T c=Q T$ when $R R=1$ :

Model 1: Linear model

$$
Q T c=Q T+\alpha(1-R R)
$$

Model 2: Hyperbolic model

$$
Q T c=Q T-\alpha(1 / R R-1)
$$

Model 3: Parabolic model

$$
Q T c=Q T / R R^{\alpha}
$$

Model 4: logarithmic model

$$
Q T c=Q T-\alpha \ln (R R)
$$

Model 5: Shifted logarithmic model

$$
Q T c=\ln \left(e^{Q T}+\alpha(1-R R)\right)
$$

Model 6: Exponential model

$$
Q T c=Q T-\alpha\left(e^{-R R}-1 / e\right)
$$

We propose in this paper to use Box-Cox transformation model to fit the QT-RR relationships, and develop a new method for correcting QT interval for heart rate. We will show that the above six parametric models are the special cases of Box-Cox model. We will assess performances of Box-Cox correction formulae using four off-drug ECG datasets and one ondrug ECG dataset, as well as a simulated dataset.

\section{Methods}

\subsection{Box-Cox approach to QT correction}


The simple linear regression model $Y=\beta_{0}+\beta X+\varepsilon$ assumes that the dependent $\mathrm{Y}$ has linear relationship with plus a random error component . This linear parametric approach can be successful provided the assumed model is appropriate. When the relationship between the dependent and independent variable is unknown or inexact, linear parametric regression can yield erroneous and even misleading results (Friedman and Stuetzle, 1981).

Instead of imposing preconceived models, we seek insight into the nature of relationships in the data set and, if possible, the underlying phenomena that might have produced the observed data values. The objective of fully exploring and explaining the effect of covariate on a dependent variable in regression analysis is facilitated by properly transforming the dependent variable and independent variable. A number of parametric transformations for continuous variables in regression analysis have been suggested (Tukey, 1957; Box and Tidwell, 1962; Box and Cox, 1964; Carroll and Ruppert, 1988; Wang and Murphy, 2004). Tukey (1957) introduced a family of parametric power transformations such that the transformed values are a monotonic function of the observed $Y$ :

$$
Y^{(\lambda)}=\left\{\begin{array}{ccc}
Y^{\lambda} & \text { if } & \lambda \neq 0 \\
\ln (Y) & \text { if } & \lambda=0
\end{array}\right.
$$

where $Y>0$. This family was later modified by Box and Cox (1964) to take account of the discontinuity at $\lambda=0$, so that the transformed values are a monotonic function of the observations,

$$
Y^{(\lambda)}=\left\{\begin{array}{lll}
\frac{Y^{\lambda}-1}{\lambda} & \text { if } & \lambda \neq 0 \\
\ln (Y) & \text { if } & \lambda=0
\end{array}\right.
$$

and for the unknown parameter $\lambda$,

$$
Y^{(\lambda)}=\beta_{0}+\beta X+\varepsilon
$$

This transformation may provide approximately normally distributed errors and make the variance more nearly constant over data points of . Substantial research has been conducted on the theoretical aspects of Box-Cox transformation and a review of this topic was provided by Sakia (1992).

Box-Cox transformation can be extended to transform both dependent variable and independent variable as follows:

$$
Y^{(\theta)}=\beta_{0}+\beta X^{(\lambda)}+\varepsilon
$$

where $Y^{(\theta)}$ and $X^{(\lambda)}$ are the transformed dependent and independent variables, respectively, and represents the random errors.

The key to the application of Box-Cox transformation technique is to estimate the transformation parameter $\theta$ and $\lambda$. Box-Cox (1964) proposed two approaches: Maximum 
likelihood approach and Bayesian approach. The maximum likelihood approach is commonly used as it is conceptually easy and the profile likelihood function is easy to compute.

In this paper, we propose using the Box-Cox transformation to fit the QT-RR relationship so that we can derive the corresponding correction formulae. We describe next four types of Box-Cox transformations following the terminology used in the Stata (StataCorp, 2009).

\section{Left-hand-side-only Box-Cox model}

The most common application of the Box-Cox transformation in linear regression analysis is to transform the dependent variable only using the parameter $\theta$. As the dependent variable is on the left-hand side of the linear regression equation, this model is known as the left-handside-only Box-Cox model (we omit random error term for the convenience of expression):

$$
Q T^{(\theta)}=\beta+\alpha R R
$$

After estimating the parameters $\theta, \alpha$ and $\beta$ in the equation (2.1), we can then derive the corresponding $Q T$ correction formulae. Considering that $Q T c$ interval represents the $Q T$ interval at a standardised heart rate of $60 /$ minute (or $Q T c=Q T$ when $R R=1$ ), the corresponding $Q T$ correction formulae can be written as:

$$
\begin{array}{ll}
Q T c=\left[Q T^{\theta}+\alpha \theta(1-R R)\right]^{1 / \theta} & \text { if } \theta \neq 0 \\
Q T c=Q T \exp [\alpha(1-R R)] & \text { if } \theta=0
\end{array}
$$

\section{Right-hand-side-only Box-Cox model}

The second model leaves the dependent variable untransformed and transforms the independent variable RR only using the parameter $\lambda$, forming a right-hand-side-only Box-Cox model. In this model, the dependent variable, QT, is expressed as:

$$
Q T=\beta+\alpha R R^{(\lambda)}
$$

The resulting correction formula from (2.2) can be shown as:

$$
\begin{array}{ll}
Q T c=Q T+\frac{\alpha}{\lambda}\left(1-R R^{\lambda}\right) & \text { if } \lambda \neq 0 \\
Q T c=Q T-\alpha \ln (R R) & \text { if } \lambda=0
\end{array}
$$

\section{Lambda model}


The third model, called Lambda model, performs the same transformation on both the dependent variable QT and independent variable RR using the parameter $\lambda$ :

$$
Q T^{(\lambda)}=\beta+\alpha R R^{(\lambda)}
$$

Using the relationship $\mathrm{QTc}=\mathrm{QT}$ when $\mathrm{RR}=1$ in equation (2.3), we have the following correction formula:

$$
\begin{array}{ll}
Q T c=\left[Q T^{\lambda}+\alpha\left(1-R R^{\lambda}\right)\right]^{1 / \lambda} & \text { if } \lambda \neq 0 \\
Q T c=Q T / R R^{\alpha} & \text { if } \lambda=0
\end{array}
$$

\section{Theta model}

The most general model is to transform the dependent variable and independent variable differently.

$$
Q T^{(\theta)}=\beta+\alpha R R^{(\lambda)}
$$

Where the dependent variable, QT, is transformed by a Box-Cox model with parameter $\theta$, and the independent variable, $R R$, is subject to a Box-Cox transformation with parameter $\lambda$. We call this model (2.4) as the Theta model.

Once the parameters $\theta, \lambda, \alpha$ and $\beta$ are estimated, we can derive a correction formula so that $Q T c=Q T$ when $R R=1$. The derived correction formula can be shown as:

$$
\begin{array}{lr}
Q T c=\left[Q T^{\theta}+\frac{\alpha \theta}{\lambda}\left(1-R R^{\lambda}\right)\right]^{1 / \theta} & \text { if } \theta \neq 0 \text { and } \lambda \neq 0 \\
Q T c=\left[Q T^{\theta}-\alpha \theta \ln (R R)\right]^{1 / \theta} & \text { if } \theta \neq 0 \text { and } \lambda=0 \\
Q T c=Q T \exp \left[\frac{\alpha}{\lambda} \ln \left(1-R R^{\lambda}\right)\right] \text { if } \theta=0 \text { and } \lambda \neq 0 \\
Q T c=Q T / R R^{\alpha} & \text { if } \theta=0 \text { and } \lambda=0
\end{array}
$$

It can be shown that Models 1, 2, 3, and 4 described in Section 1 are special cases of BoxCox model. For example,

when $\theta=1$ and $\lambda=1$, we have $Q T c=Q T+\alpha(1-R R)$, linear model (Model 1);

when $\theta=1$ and $\lambda=-1$, we have $Q T c=Q T+\alpha(1-1 / R R)$, hyperbolic model (Model 2);

when $\theta=0$ and $\lambda=0$, we have $Q T c=Q T / R R^{\alpha}$, parabolic model (Model 3);

when $\theta=1$ and $\lambda=0$, we have $Q T c=Q T-\alpha \ln (R R)$, loagrithmic model (Model 4); 
For Models 5 and 6 , although there are no specific $\theta$ and $\lambda$ values that can be used to express explicit relationships, their correction formulae could be approximated by the Box-Cox model. For example, the Model $5 Q T=\ln (\beta+\alpha R R) \operatorname{or} \exp (Q T)=\beta+\alpha R R$, can be approximated by the left-hand-side-only Box-Cox model $\frac{Q T^{\theta}-1}{\theta}=\beta+\alpha R R$. If we could find the $\theta$ that satisfies the relationship $\exp (Q T)=\frac{Q T^{\theta}-1}{\theta}$, we can then establish that Model 5 is a special case of lefthand-side-only Box-Cox model. There is no explicit expression for $\theta$ and finding out $\theta$ needs numerical calculations.

A Box-Cox correction formula can be derived in three steps and we demonstrate the procedures using the Theta model. The first step is to estimate the parameters $\theta$ and $\lambda$ in a BoxCox model by the maximum likelihood method. This can be done using Stata (StataCorp, 2009). The second step is to calculate the Box-Cox transformed $Q T$ (i.e. $Q T^{(\theta)}$ ) and $R R$ (i.e. $R R^{(\lambda)}$ ) using the parameter derived in Step 1. In the third step, the correction factor $\alpha$ is determined using the simple least square linear regression model $Q T^{(\theta)}=\beta+\alpha R R^{(\lambda)}$. Introducing the estimated parameters $\theta, \lambda, \alpha$ into $Q T c=\left[Q T^{\theta}+\frac{\alpha \theta}{\lambda}\left(1-R R^{\lambda}\right)\right]^{1 / \theta}$ gives the QTc formula under the Theta model.

\subsection{Assessment of the performances of Box-Cox QT correction methods}

To assess the performances of the four correction formulae, we use the following five measurements.

For regression models, the Root Mean Squared Error (RMSE) is often used to assess the goodness-of-fit of various QT-RR relationship models. The RMSE is defined as

$$
R M S E=\sqrt{\frac{\sum_{i=1}^{n}\left(y_{i}-\hat{y}_{i}\right)^{2}}{n}}
$$

A smaller RMSE value indicates a better model fitting but does not necessarily mean a better QT correction formula. The purpose of a correction formula is to generate QTc values that are uncorrelated with RR intervals. Pearson correlation coefficient $(\rho)$ between QTc and $\mathrm{RR}$ is often used to assess if there is a linear relationship between the QTc and RR. If $\boldsymbol{\rho}$ is zero, QTc is considered to be independent of RR (Bazett, 1920; Malik et al., 2002; Camm and Malik, 2002; Wang et al., 2010). A correlation of 0 means there is no linear relationship between the 
two variables. However, zero correlation coefficient between QTc and RR does not necessarily mean their statistical independence if the relationship between QTc and RR is non-linear (Wang et al., 2010). Therefore, the slope from regression of QTc on RR has been used to assess if there is non-linear correlation between QTc and RR (Desai et al., 2003), and the slope of QTc-RR regression is expected to be zero when QTc values are independent of RR values.

A correction formula would be much preferable in design of a thorough QT study if its resulting QTc intervals are less variable (Desai et al., 2003; Malik et al., 2004; Zhang and Machado, 2008; Tsong et al., 2008; Wang et al., 2012). For example, a smaller sample size would be needed if the standard deviation of QTc is reduced. We therefore use two measurements of QTc variation: standard deviation and range.

\section{Results}

\subsection{Example 1. Randomised Clinical Trials Data}

Our first example is a sample from 4 thorough QT trials conducted at Richmond Pharmacology from 2007 to 2008. In these trials, the ECG databases were derived from the collection of standard 12-lead surface ECGs performed during the trials. In order to address intrinsic variability of the QT/QTc interval, which can be influenced by many factors including activity level, postural changes, circadian patterns, emotional state or food ingestion, the ECGs were collected at multiple times using 12 lead ECG recordings. At each time point, the ECGs were recorded in triplicate, to reduce variance and improve the precision of measurement. Each ECG lasted 10 seconds and the triplicates were performed at 1-minute intervals. Before any ECG recording, the subjects maintained an undisturbed supine resting position for at least 10 minutes. The subjects were asked to avoid postural changes during the ECG recordings.

In this example, we used the baseline ECGs which were not affected by study drugs. This is a common practice in thorough QT trials in order to derive the individual correction formula (Malik 2001; ICH, 2005). A total 38,467 ECGs were used in this example and these ECGs were contributed by 225 healthy volunteers aged between 20 and 50 years. The ranges for QT and RR were 0.310-0.498 seconds and 0.521-1.667 seconds, respectively.

Table 1 presents mean values of various measurements of QT correction performance over 225 subjects by four Box-Cox models with their correction factors. The absolute values of correlation coefficient and regression slope of QTc-RR were used for calculating their mean values. Several observations can be made from this table. An optimum case is the model with the smallest value out of the four models for a subject's ECG profile.

Firstly, there is no universally applicable correction formula that produces consistently best performance in terms of the five measurements for assessing QT correction formulae as reflected in the number of optimum cases. This suggests that the best QT correction is often data driven: a correction method may work better for one ECG profile than another.

Secondly, the values of the mean absolute correlation coefficient and the mean absolute regression slope of QTc-RR are close to zero for all four QT correction formulae. This suggests 
that the independence between QTc and RR, the primary objective of QT correction, can be achieved using the QT correction formulae derived from Box-Cox transformations.

Thirdly, although the 'Right hand-sided only model' as described in equation (2.2) has the smallest mean values for RMSE, $\boldsymbol{\rho}$ and slope, the Theta model in equation (2.4) has the smallest mean values for standard deviation and range of QTc.

Fourthly, the 'Right hand-sided only model' generates the smallest variability in the correction factor $\alpha$ whereas the Lambda model in (2.3) and Theta model in (2.4) yield the QTc with very large standard variation of 434.106 and 649.531, respectively, due to some extreme values of $\theta$ and $\lambda$ for some subjects.

\subsection{Example 2. Simulation Study}

In this example, we use the simulated dataset to assess the performance of four Box-Cox models. The aim of the simulation is to get a wide range of possible QT-RR patterns. First, we randomly selected 10 subjects from 225 subjects in Example 1 and then randomly selected 10\% of ECG data from each of 10 subjects to form a new subject. This strategy was also used by Malik et al. (2004) in their simulation studies. This process is independently repeated 1000 times, generating a new sample of 1000 subjects.

Table 2 presents the mean values of various measurements of QTc by four different models using the simulated dataset. The simulation results are generally in agreement with those observed in Table 1. However, the variation in correction factor $\alpha$ for all four models becomes smaller compared with that in Table 1 . This is because ranges of $\theta$ and $\lambda$ estimated from newly simulated datasets become smaller than those from the original 225 subjects.

\subsection{Example 3. 4-way Crossover Study}

Our third example is a single-centre, randomised, placebo-controlled, double blind, $4 \times 4$ crossover trial in 64 healthy volunteers who were randomised into a 4 period crossover comparison of placebo, moxifloxacin and levofloxacin. Twelve-lead ECGs were recorded using a MAC1200 ${ }^{\circledR}$ recorder (GE Healthcare) and stored electronically on the MUSE $\mathrm{CV}^{\circledR}$ information system (GE Healthcare). This trial has 24,893 ECGs in total, of which 14,241 are off-drug ECGs and 10,652 on-drug ECGs.

This example is different from Example 1 in that it not only contains the off-drug ECGs but also on-drug ECGs, enabling us to evaluate various QT correction methods under the real-life scenario of a thorough QT study. To analyse this dataset, we first estimated the individual correction factors $\alpha$ using the 14,241 off-drug ECGs at baseline and then applied the individual correction formulae to both off-drug and on-drug ECGs and calculated mean values of various measurements of QT correction evaluation for 64 subjects in this study (Table 3).

The results in Table 3 are generally consistent with those observed from Example 1 and 2. In particular, all four QT correction formulae derived from the Box-Cox transformation yield 
QTc that are independent of RR, and QTc from the Theta model and Lambda model have smaller variability.

\section{Discussion}

QT interval is an important parameter of surface resting ECG and is used as a surrogate endpoint in drug development to detect the potential of drugs to cause life threatening cardiac arrhythmias (Malik 2001; ICH, 2005; Goldenberg et al., 2006). As a result, the assessment of drug-induced QT interval prolongation has attracted significant attention from both pharmaceutical companies and the regulatory authorities (Malik 2001; ICH, 2005).

Box-Cox transformation has been widely used in medical research since it was first proposed (Box and Cox, 1964; Carroll and Ruppert, 1988). In this study, we propose to use the Box-Cox transformation to fit the QT-RR relationships in order to derive new QT correction formulae. Box-Cox model represents a parametric family for fitting a relationship between two or more variables. Box-Cox approach is particularly useful for the following situations: (1) The relationship between two variables is expected to be of a complex form and not easily characterised by standard linear or non-linear models. (2) There is no a priori reason for using a particular model. (3) One wants the data itself to suggest what the most appropriate functional form is. QT correction often involves these situations, it is therefore preferable to use Box-Cox transformation model to analyse the QT interval data. We have shown that the six commonly used QT correction models are the special cases of the Box-Cox model.

The results presented here from 225 individual QT-RR profiles from the 4 clinical trials demonstrate that the QT correction formulae derived from the Box-Cox transformations could generate the QTc independent of RR, the primary purpose of QT correction. The results from the simulated 1000 individual ECG data and on-drug ECG data confirmed the above observation. Furthermore, our analyses demonstrate that the Theta model and Lambda models generate QTc with smaller variations. This is a desirable property because a smaller variation in QTc means more precise estimate (a narrower interval) of treatment effect. The Theta and Lambda models include the parabolic model $\mathrm{QT}=\beta \mathrm{RR} \alpha$ (the Bazzet and Fridericia correction are the special cases of the parabolic model) (Bazett, 1920; Fridericia, 1920). This finding is consistent with Wang et al study (2012).

Over the last several decades, many authors have attempted to find a "true" and universally applicable correction formula, assuming that the investigated QT and RR relationship is representative of a "physiological" mechanism that is the same in every healthy subject or at least in a same subject of a well-defined population (e.g., healthy men) (Malik, 2001; Malik, 2002). Empirical results have appeared to demonstrate that such attempts are futile (Malik, 2001; Malik, 2002; Wang et al., 2012). Our results also confirm this observation and show that there is no universally applicable correction formula that produces consistently better performance in terms of all five measurements for assessing QT correction formulae. 


\section{Conclusions}

The Box-Cox transformation represents a very flexible family for modelling QT-RR relationships including the six commonly used parameter models, thus providing a potentially better QT correction method than the existing parametric models. Furthermore, the implementation of Box-Cox transformation proposed in this study can be easily implemented through Stata software. Lastly, although all four QT correction formulae derived from the BoxCox transformation generate QTc independent of heart rate RR, the Box-Cox transformation of both QT and RR generates QTc with smaller variations.

\section{Author contributions}

DW designed the study, performed data analysis and interpret the results and drafted the article. RA and JT reviewed the article.

\section{References}

[1] Bazett, J.C. (1920). An analysis of time relations of electrocardiograms. Heart 7:353-367.

[2] Box, G.E.P. and Cox, D.R. (1964). An analysis of transformations. Journal of the Royal Statistical Society Series B 26: 211-252.

[3] Box, G.E.P. and Tidwell, P.W. (1962). Transformation of independent variables. Technometrics 4:531-550.

[4] Camm, A.J. and Malik, M. (2002). QT-RR relationship in healthy subjects exhibits substantial intersubject variability and high intrasubject stability. American Journal of Physiology 282:2356-2363

[5] Carroll, R.J. and Ruppert, D. (1988). Transformation and Weighting in Regression New York: Chapman and Hall.

[6] Desai, M., Li, L., Desta, Z., Malik, M., Flockhart, D. (2003). Variability of heart rate correction methods for the QT interval: potential for QTc overestimation with a low dose of haloperidol. British Journal of Clinical Pharmacology 55(6):511-517.

[7] Fridericia, L.S. (1920). Die Systolendauer im Elekrokardiogramm bei normalen Menschen und bei Herzkranken. Acta Medica Scandinavica 53:469- 486.

[8] Friedman, J.H. and Stuetzle, W. (1981). Projection pursuit regression. Journal of the American Statistical Association 76(376):817-823. 
[9] Goldenberg, I., Moss, A.J., Zareba, W. (2006). QT Interval: How to Measure It and What Is "Normal”. Journal of Cardiovascular Electrophysiology 17(3):333-336.

[10] Hodges, M., Salerno, D., Erlien, D. (1983). Bazzet's QT correction reviewed-evidence that a linear QT correction for heart is better. Journal of the American College of Cardiology 1(3):694.

[11] ICH (2005). ICH E14 Guidance: The Clinical Evaluation of QT/QTc Interval Prolongation and Proarrhythmic Potential for Nonantiarrhythmic Drugs. International Conference on Harmonization, Geneva, Switzerland.

[12] Malik, M. (2001). Problems of heart rate correction in assessment of drug-induced QT interval prolongation. Journal of Cardiovascular Electrophysiology 12:411- 420.

[13] Malik, M. (2002). Is there a physiologic QT/RR relationship? Journal of Cardiovascular Electrophysiology 13(12):1219-21

[14] Malik, M., Färbom, P., Batchvarov, V., Hnatkova, K., Camm, A.J. (2002). Relation between QT and RR intervals is highly individual among healthy subjects: Implications for heart rate correction of the QT interval. Heart 87:220-228.

[15] Malik, M., Hnatkova, K., Batchvarov, V. (2004). Differences between study-specific and subject specific heart rate corrections of the QT interval in investigations of drug-induced QTc prolongation. PACE 27: 791-800.

[16] Sagie, A., Larson, M.G., Goldberg, R.J., Bengtson, J.R. and Levy, D. (1992). An improved method for adjusting the QT interval for heart rate (the Framingham heart study). American Journal of Cardiology 70: 797-801.

[17] Sakia, R.M. (1992). The Box-Cox transformation technique: a review. The Statistician 41,169-178.

[18] StataCorp (2009). Stata 11 Base Reference Manual. College Station, TX: Stata Press.

[19] Tsong, Y., Shen, M., Zhong, J., Zhang, J. (2008). Statistical issues of QT prolongation assessment based on linear concentration modeling. Journal of Biopharmaceutical Statistics 18(3):564-84

[20] Tukey, J.W. (1957). The comparative anatomy of transformation. Annals of Mathematical Statistics 28, 602-632.

[21] Wang, D., Cheung, Y., Arezina, R., Taubel, J., Camm, A.J. (2010). A Nonparametric Approach to QT Interval Correction for Heart Rate. Journal of Biopharmaceutical Statistics 20:508-522. 
[22] Wang, D. and Murphy, M. (2004). Estimating the optimal transformation for multiple regression using ACE algorithm. Journal of Data Science 3(2):23-34.

[23] Wang, D., Taubel, J., Arezina, R. (2012). Comparison of six commonly used QT correction models and their parameter estimation methods. Journal of Biopharmaceutical Statistics 22(6):1148-61.

[24] Zhang, J. and Machado, S.G. (2008). Statistical issues including design and sample size calculation in thorough QT/QTc studies. Journal of Biopharmaceutical Statistics 18(3):451-67.

Received April 15, 2015; accepted October 10, 2015.

Duolao Wang, PhD

Department of Clinical Sciences

Liverpool School of Tropical Medicine

Pembroke Place, Liverpool, L3 5QA, UK.

Duolao.Wang@1stmed.ac.uk

Table 1: Summary statistics of model parameters and measurements of QT correction performance for 225 subjects (38467 ECGs) from 4 thorough QT studies

\begin{tabular}{|c|c|c|c|c|c|c|}
\hline & & r Statistics & $\begin{array}{l}\text { Left hand } \\
\text { sided only } \\
\text { model }\end{array}$ & $\begin{array}{l}\text { Right hand } \\
\text { sided only } \\
\text { model }\end{array}$ & $\begin{array}{c}\text { Lambda } \\
\text { model }\end{array}$ & $\begin{array}{l}\text { Theta } \\
\text { model }\end{array}$ \\
\hline \multirow{10}{*}{$\begin{array}{l}\text { Model } \\
\text { parameter }\end{array}$} & \multirow[t]{5}{*}{$\theta$} & Mean & 1.334 & & -0.362 & 0.019 \\
\hline & & SD & 3.244 & & 2.175 & 3.576 \\
\hline & & Median & 1.080 & & -0.151 & -0.074 \\
\hline & & Minimum & -7.323 & & -10.393 & -10.421 \\
\hline & & Maximum & 8.868 & & 4.679 & 9.063 \\
\hline & \multirow[t]{5}{*}{$\bar{\lambda}$} & Mean & & -0.137 & -0.362 & -0.419 \\
\hline & & $\mathrm{SD}$ & & 2.156 & 2.175 & 2.321 \\
\hline & & Median & & 0.196 & -0.151 & -0.252 \\
\hline & & Minimum & & -16.198 & -10.393 & -15.777 \\
\hline & & Maximum & & 4.463 & 4.679 & 4.824 \\
\hline
\end{tabular}




\begin{tabular}{|c|c|c|c|c|c|c|}
\hline & Parameter & Statistics & $\begin{array}{l}\text { Left hand } \\
\text { sided only } \\
\text { model }\end{array}$ & $\begin{array}{l}\text { Right hand } \\
\text { sided only } \\
\text { model }\end{array}$ & $\begin{array}{c}\text { Lambda } \\
\text { model }\end{array}$ & $\begin{array}{l}\text { Theta } \\
\text { model }\end{array}$ \\
\hline \multirow{15}{*}{$\begin{array}{l}\text { Model } \\
\text { performance }\end{array}$} & \multirow[t]{5}{*}{$\alpha$} & Mean & 4.075 & 0.153 & 37.351 & 67.865 \\
\hline & & $\mathrm{SD}$ & 20.708 & 0.052 & 434.106 & 649.531 \\
\hline & & Median & 0.126 & 0.147 & 0.398 & 0.376 \\
\hline & & Minimum & 0.000 & 0.030 & 0.002 & 0.000 \\
\hline & & Maximum & 235.332 & 0.503 & 6480.573 & 9567.549 \\
\hline & \multirow[t]{2}{*}{ RMSE } & Mean & 6.989 & 6.899 & 6.933 & 6.910 \\
\hline & & $\begin{array}{l}\text { Optimum } \\
\text { cases }\end{array}$ & 3 & 174 & 19 & 34 \\
\hline & \multirow[t]{2}{*}{$\rho$} & Mean & 0.003 & 0.001 & 0.009 & 0.003 \\
\hline & & $\begin{array}{l}\text { Optimum } \\
\text { cases }\end{array}$ & 42 & 134 & 18 & 36 \\
\hline & \multirow[t]{2}{*}{ slope } & Mean & 0.000 & 0.000 & 0.001 & 0.000 \\
\hline & & $\begin{array}{l}\text { Optimum } \\
\text { cases }\end{array}$ & 42 & 135 & 18 & 35 \\
\hline & \multirow[t]{2}{*}{ QTc SD } & Mean & 7.024 & 6.924 & 6.700 & 6.725 \\
\hline & & $\begin{array}{l}\text { Optimum } \\
\text { cases }\end{array}$ & 35 & 55 & 56 & 84 \\
\hline & \multirow{2}{*}{$\begin{array}{l}\text { QTc } \\
\text { Range }\end{array}$} & Mean & 37.674 & 37.676 & 36.554 & 36.223 \\
\hline & & $\begin{array}{l}\text { Optimum } \\
\text { cases }\end{array}$ & 46 & 52 & 59 & 73 \\
\hline
\end{tabular}

RMSE=Root Mean Squared Error, SD=Standard Deviation, Range=Maximum-Minimum

Table 2: Summary statistics of model parameters and measurements of QT correction performance for 1000 simulated subjects (171048 ECGs)

\begin{tabular}{|c|c|c|c|c|c|c|}
\hline & Parameter & Statistics & $\begin{array}{l}\text { Left hand } \\
\text { sided only } \\
\text { model }\end{array}$ & $\begin{array}{l}\text { Right hand } \\
\text { sided only } \\
\text { model }\end{array}$ & $\begin{array}{c}\text { Lambda } \\
\text { model }\end{array}$ & $\begin{array}{l}\text { Theta } \\
\text { model }\end{array}$ \\
\hline \multirow{4}{*}{$\begin{array}{l}\text { Model } \\
\text { parameter }\end{array}$} & \multirow[t]{4}{*}{$\theta$} & Mean & 0.683 & & -0.422 & -0.627 \\
\hline & & SD & 1.838 & & 1.401 & 2.164 \\
\hline & & Median & 0.750 & & -0.459 & -0.735 \\
\hline & & Minimum & -5.869 & & -4.609 & -7.121 \\
\hline
\end{tabular}




\begin{tabular}{|c|c|c|c|c|c|c|}
\hline & Parameter & Statistics & $\begin{array}{l}\text { Left hand } \\
\text { sided only } \\
\text { model }\end{array}$ & $\begin{array}{c}\text { Right hand } \\
\text { sided only } \\
\text { model }\end{array}$ & $\begin{array}{c}\text { Lambda } \\
\text { model }\end{array}$ & $\begin{array}{l}\text { Theta } \\
\text { model }\end{array}$ \\
\hline & & Maximum & 6.927 & & 5.342 & 8.523 \\
\hline & $\lambda$ & Mean & & 0.128 & -0.422 & -0.404 \\
\hline & & $\mathrm{SD}$ & & 1.234 & 1.401 & 1.428 \\
\hline & & Median & & 0.085 & -0.459 & -0.480 \\
\hline & & Minimum & & -3.782 & -4.609 & -5.046 \\
\hline & & Maximum & & 4.391 & 5.342 & 4.391 \\
\hline & $\alpha$ & Mean & 0.751 & 0.146 & 1.130 & 3.559 \\
\hline & & $\mathrm{SD}$ & 2.467 & 0.021 & 1.882 & 12.420 \\
\hline & & Median & 0.180 & 0.147 & 0.553 & 0.671 \\
\hline & & Minimum & 0.001 & 0.080 & 0.002 & 0.000 \\
\hline & & Maximum & 55.483 & 0.211 & 23.097 & 228.545 \\
\hline \multirow{10}{*}{$\begin{array}{l}\text { Model } \\
\text { performance }\end{array}$} & RMSE & Mean & 12.797 & 12.497 & 12.571 & 12.522 \\
\hline & & $\begin{array}{l}\text { Optimum } \\
\text { cases }\end{array}$ & 18 & 764 & 67 & 164 \\
\hline & $\rho$ & Mean & 0.004 & 0.002 & 0.014 & 0.005 \\
\hline & & $\begin{array}{l}\text { Optimum } \\
\text { cases }\end{array}$ & 213 & 618 & 51 & 131 \\
\hline & slope & Mean & 0.000 & 0.000 & 0.001 & 0.000 \\
\hline & & $\begin{array}{l}\text { Optimum } \\
\text { cases }\end{array}$ & 211 & 614 & 52 & 136 \\
\hline & QTc SD & Mean & 12.614 & 12.534 & 12.341 & 12.157 \\
\hline & & $\begin{array}{l}\text { Optimum } \\
\text { cases }\end{array}$ & 87 & 216 & 230 & 480 \\
\hline & QTc & Mean & 65.424 & 64.843 & 63.307 & 62.822 \\
\hline & Range & $\begin{array}{l}\text { Optimum } \\
\text { cases }\end{array}$ & 172 & 197 & 286 & 358 \\
\hline
\end{tabular}

RMSE=Root Mean Squared Error, SD=Standard Deviation, Range=Maximum-Minimum

Table 3: Summary statistics of model parameters and measurements of QT correction performance for 64 subjects from a $4 \times 4$ crossover trial (24546 ECGs) 


\begin{tabular}{|c|c|c|c|c|c|c|}
\hline & Paramete & r Statistics & $\begin{array}{l}\text { Left hand } \\
\text { sided only } \\
\text { model }\end{array}$ & $\begin{array}{l}\text { Right hand } \\
\text { sided only } \\
\text { model }\end{array}$ & $\begin{array}{c}\text { Lambda } \\
\text { model }\end{array}$ & $\begin{array}{l}\text { Theta } \\
\text { model }\end{array}$ \\
\hline \multirow{15}{*}{$\begin{array}{l}\text { Model } \\
\text { parameter }\end{array}$} & \multirow[t]{5}{*}{$\theta$} & Mean & 1.388 & & -0.716 & -0.221 \\
\hline & & SD & 3.346 & & 2.391 & 3.761 \\
\hline & & Median & 1.444 & & -0.567 & -0.249 \\
\hline & & Minimum & -5.835 & & -6.290 & -8.871 \\
\hline & & Maximum & 7.505 & & 4.679 & 7.459 \\
\hline & \multirow[t]{5}{*}{$\lambda$} & Mean & & -0.410 & -0.716 & -0.722 \\
\hline & & SD & & 2.146 & 2.391 & 2.470 \\
\hline & & Median & & -0.501 & -0.567 & -0.425 \\
\hline & & Minimum & & -6.595 & -6.290 & -7.182 \\
\hline & & Maximum & & 3.829 & 4.679 & 4.824 \\
\hline & \multirow[t]{5}{*}{$\alpha$} & Mean & 2.819 & 0.151 & 10.763 & 57.308 \\
\hline & & $\mathrm{SD}$ & 10.943 & 0.059 & 35.253 & 226.511 \\
\hline & & Median & 0.100 & 0.139 & 0.496 & 0.387 \\
\hline & & Minimum & 0.000 & 0.074 & 0.004 & 0.000 \\
\hline & & Maximum & 82.940 & 0.490 & 207.546 & 1369.067 \\
\hline \multirow{10}{*}{$\begin{array}{l}\text { Model } \\
\text { performance }\end{array}$} & \multirow[t]{2}{*}{ RMSE } & Mean & 6.190 & 6.103 & 6.138 & 6.112 \\
\hline & & $\begin{array}{l}\text { Optimum } \\
\text { cases }\end{array}$ & 2 & 47 & 4 & 11 \\
\hline & \multirow[t]{2}{*}{$\rho$} & Mean & 0.003 & 0.001 & 0.010 & 0.002 \\
\hline & & $\begin{array}{l}\text { Optimum } \\
\text { cases }\end{array}$ & 10 & 39 & 4 & 11 \\
\hline & \multirow[t]{2}{*}{ slope } & Mean & 0.000 & 0.000 & 0.001 & 0.000 \\
\hline & & $\begin{array}{l}\text { Optimum } \\
\text { cases }\end{array}$ & 10 & 40 & 4 & 10 \\
\hline & \multirow[t]{2}{*}{ QTc SD } & Mean & 6.072 & 6.117 & 5.782 & 5.736 \\
\hline & & $\begin{array}{l}\text { Optimum } \\
\text { cases }\end{array}$ & 12 & 11 & 20 & 21 \\
\hline & QTc & Mean & 34.905 & 36.207 & 34.203 & 32.907 \\
\hline & Range & $\begin{array}{l}\text { Optimum } \\
\text { cases }\end{array}$ & 11 & 9 & 19 & 25 \\
\hline
\end{tabular}

RMSE=Root Mean Squared Error, $\mathrm{SD}=$ Standard Deviation, Range=Maximum-Minimum 